\title{
A Comparative Study on Optimal Structural Dynamics Using Wavelet Functions
}

\author{
Seyed Hossein Mahdavi and Hashim Abdul Razak \\ StruHMRS Group, Department of Civil Engineering, University of Malaya, 50603 Kuala Lumpur, Malaysia \\ Correspondence should be addressed to Hashim Abdul Razak; hashim@um.edu.my
}

Received 2 April 2014; Accepted 8 October 2014

Academic Editor: Shuenn-Yih Chang

Copyright ( $) 2015$ S. H. Mahdavi and H. Abdul Razak. This is an open access article distributed under the Creative Commons Attribution License, which permits unrestricted use, distribution, and reproduction in any medium, provided the original work is properly cited.

\begin{abstract}
Wavelet solution techniques have become the focus of interest among researchers in different disciplines of science and technology. In this paper, implementation of two different wavelet basis functions has been comparatively considered for dynamic analysis of structures. For this aim, computational technique is developed by using free scale of simple Haar wavelet, initially. Later, complex and continuous Chebyshev wavelet basis functions are presented to improve the time history analysis of structures. Free-scaled Chebyshev coefficient matrix and operation of integration are derived to directly approximate displacements of the corresponding system. In addition, stability of responses has been investigated for the proposed algorithm of discrete Haar wavelet compared against continuous Chebyshev wavelet. To demonstrate the validity of the wavelet-based algorithms, aforesaid schemes have been extended to the linear and nonlinear structural dynamics. The effectiveness of free-scaled Chebyshev wavelet has been compared with simple Haar wavelet and two common integration methods. It is deduced that either indirect method proposed for discrete Haar wavelet or direct approach for continuous Chebyshev wavelet is unconditionally stable. Finally, it is concluded that numerical solution is highly benefited by the least computation time involved and high accuracy of response, particularly using low scale of complex Chebyshev wavelet.
\end{abstract}

\section{Introduction}

Generally, results integrated from dynamic analysis of structures are the main reliable criteria for design of solids and structures. The technique for a solution of general dynamic equilibrium can become very expensive if a complex loading (e.g., an unknown earthquake excitation) is being applied on large-scaled structures $[1,2]$. In general, procedure of dynamic analysis is categorized into the two solution methods: first, mode superposition method. Second, direct and indirect integration methods, for example, central differences, family of Newmark- $\beta$, Houbolt, and Wilson- $\theta$ fall under direct integration schemes and Fourier transformation and wavelet analysis have been introduced as indirect integration approaches. In addition, the choice as to which method to use for an effective solution is governed by the dynamical problem considered [3,4]. Theoretically, aforesaid numerical integration methods lie in case of either conditionally stable or unconditionally stable procedures. An integration method is unconditionally stable if the solution for any initial conditions does not grow without a band for any time step while will be conditionally stable if it does grow. In other words, stability of results is achieved at any time interval and there is no restriction on using $\Delta t$ smaller than a particular value. As a result, optimal solution for dynamic analysis is accomplished using long intervals [5-7].

Nowadays, orthogonal polynomials are being widely implemented as a practical analysis of time dealing problems in engineering, particularly in the form of wavelet analysis. Obvious effectiveness from property of orthogonality is that the repeated components with similar characteristics are neglected in the analytical process $[8,9]$. Consequently, computational calculations are being considerably decreased and computation time involved is being therefore reduced, hence, accuracy of responses will be more desirable [10, 11]. Practically, considerable attention has been given for the use of wavelet method for the solution of time dependent problems such as dynamic analysis or identification of systematic 
problems [12]. Several attractive mathematical characteristics of wavelets such as efficient multiscale decompositions, localization properties in physical and wave-number spaces, and the existence of recursive and fast wavelet transforms have obtained practice of this efficient tool for the numerical solution of partial differential equations (PDEs) and ordinary differential equations (ODEs) [13-15].

Significantly, accuracy of responses is directly related to the basis function of mother wavelet, depending on the kind of objectives. Fundamentally, in the structural dynamics, compatibility of a wavelet basis function is premised upon on not only the degrees of freedom but also the similarity of basic functions to the lateral loading, emphasizing on frequency contents. Considerably, less computational cost of calculations in advanced time history analysis through the compatible wavelet functions makes distinction of this approach over other numerical methods. For instance, for the purpose of time history analysis, a simple basis function of Haar wavelet was indirectly applied on its own freescaled functions [16]. It is inferred that because of the inherent simple shape function of Haar wavelet accuracy of responses was undesirable even employing large-scaled functions. Furthermore, to improve inadequacy of Haar wavelet known as the simplest and two-dimensional (2D) wavelet basis function, it is indispensable to employ threedimensional (3D) and adaptive wavelet basis functions [17, 18]. Moreover, there is no consideration reported on the stability of responses calculated using Haar wavelet functions compared with other basis functions.

Mathematically, adaptive wavelets are those that grow in three dimensions, which in the current definition dimensions are time, scale, and frequency, respectively. For instance, Chebyshev, Legender, and Symlet are some basis functions with compatible characteristics [19]. Basically, Chebyshev polynomials are presented as continuous basis function of wavelet $[20,21]$. The most popular characteristic of this wavelet is various weight functions of Chebyshev polynomials that directly influence stability and accuracy of responses [22-24]. However, it is reported that stability of results computed by Chebyshev wavelet are independent from initial accelerations. Furthermore, compatibility is being satisfied through the capturing of broad frequency of complex excitations by oscillated shape functions of free-scaled Chebyshev wavelet [25].

Subsequently, the main contributions of this study (which received little attention in the literature) are composed of the following: (i) a numerical assessment of structural dynamic problems using free-scaled simple and complex wavelet functions, with the emphasis on the large scales of wavelets, (ii) numerical stability analysis of an indirect algorithm using family of discrete Haar wavelets, established as 2D wavelet functions, (iii) stability analysis of continuous Chebyshev wavelet functions with respect to the thirdordered derivation of time, (iv) a comparative evaluation of computational efficiency of simple and complex wavelets for smooth and wide-band frequency contents of loading, and (v) capability evaluation of Haar and Chebyshev wavelets in linear and nonlinear structural dynamic problems. Accordingly, to achieve the proposed objectives of this paper, the second-ordered differential equation of motion is indirectly solved by free scale of Haar wavelet and later, freescaled Chebyshev wavelet functions are directly implemented to compute responses. For this aim, a brief background of wavelet is discussed in Section 2 of this study. In addition, coefficient matrices of wavelet and operation matrices of integration corresponding to complex scales of efficient Chebyshev wavelet are formulated and presented in this section. In Section 3, the computational procedure is developed for an optimal dynamic analysis. Section 4 is allocated to numerical stability analysis of responses, corresponding to the indirect method proposed for Haar and direct scheme proposed for Chebyshev wavelet functions. Accordingly, stability of responses has been comparatively presented. Section 5 is devoted to investigation of the validity and effectiveness of results. For this purpose, various scales of Chebyshev and Haar wavelet functions are considered for dynamic analysis. Finally, efficiency and accuracy of results have been compared in this section.

\section{Fundamental of Wavelet}

2.1. Haar Wavelet. The simple family of Haar wavelet was presented by Alfred Haar in 1910 for $t \in[0,1]$ as follows $[16,18]$ :

$$
H_{m-1}(t)= \begin{cases}1, & t \in\left[\frac{k}{2^{j}}, \frac{k+0.5}{2^{j}}\right], \\ -1, & t \in\left[\frac{k+0.5}{2^{j}}, \frac{k+1}{2^{j}}\right], \\ 0, & \text { otherwise, }\end{cases}
$$

where

$$
m=2^{j}+b+1, \quad j \geq 0,0 \leq b \leq 2^{j}-1,
$$

where $M=2^{j}(j=0,1, \ldots, j)$ denotes the order of wavelet; $b=0,1, \ldots, M-1$ is the value of transition. In (1), $m=1$ and $m=2$ indicate scale function and mother wavelet of Haar, respectively. In this study, $2 M$ indicates the number of segmentations in each global time interval regarding the scale of proposed wavelet which refers to segmentation method (SM). For example, in the case of Haar wavelet $2 M=2^{j+1}$ denotes the $2^{j}$ th scale of Haar wavelet [18].

Basically, signal $f(t)$ can be expanded in Haar series as $[16]:$

$$
f(t) \cong \sum_{i=0}^{2 M} c_{i} h_{i}(t) .
$$

Accordingly, Haar coefficients $c_{i}(i=0,1,2, \ldots)$ are defined by

$$
c_{i}=2^{j} \int_{0}^{1} f(t) h_{i}(t) d t .
$$

Hence, $H_{2 M}$ is a square matrix $(2 M \times 2 M)$, including the first $2 M$ scale of Haar wavelet; Haar coefficients are directly given as

$$
c_{i}=f(t) H_{2 M}^{-1}(t)
$$


Equivalently, signal $f(t)$ may be rewritten as

$$
f(t) \cong c_{2 M}^{T} H_{2 M}(t) .
$$

Subsequently, integration of $H_{2 M}$ is obtained by Haar series with new square coefficient matrix of integration $P_{2 M}$ as $[16,18]$

$$
\int_{0}^{1} H_{2 M}(t) d t \approx P H_{2 M}(t)
$$

Pointed out that local times are calculated relatively to the scale of wavelet as

$$
\tau_{l}=\frac{l-0.5}{2 M}, \quad l=1,2, \ldots, 2 M
$$

Finally, the local time divisions $\left(\tau_{l}\right)$ are adapted to the global domain. Assumption of $d_{t}$ as global time interval we have $[16,18]$

$$
t_{l t}=d_{t}\left(\tau_{l}\right)+t_{t} \Longrightarrow \tau_{l}=\frac{t_{t}-t_{l t}}{d_{t}}, \quad l=1,2, \ldots, 2 M .
$$

2.2. Chebyshev Wavelet. In mathematics, families of continuous wavelet transforms (CWT) are considered as follows $[22,24]$ :

$$
\begin{aligned}
& \text { CWT (Scale, Position) } \\
& =\int f(t) \Psi(\text { Scale, Position) } d t,
\end{aligned}
$$

where CWT denotes corresponding wavelet transform. As long as wavelet function $\Psi(t)$ is supposed as mother wavelet, the continuous wavelet transform of a signal $f(t)$ is obtained as

$$
\operatorname{CWT}_{S}^{\Psi}(a, b)=\frac{1}{\sqrt{|a|}} \int f(t) \Psi_{a, b}^{*}(t) d t
$$

where $b, a$ indicate the transition and the scale of wavelet and * indicates the complex conjugate form of $\Psi(t)$, respectively.

In general, the Chebyshev polynomials, named after Pafnuty Chebyshev, are a sequence of orthogonal polynomials defined as two kinds. The general expression for Chebyshev polynomials of the first kind $\left(T_{n}\right)$ is defined as follows $[20,21]$ :

$$
\begin{array}{r}
T_{n}(x) \\
=\left(\frac{n}{2}\right) \sum_{k=0}^{n / 2}\left((-1)^{k} \frac{n-k-1 !}{n-2 k ! k !} \times(2 x)^{n-2 k}\right), \\
n=1,2,3, \ldots .
\end{array}
$$

In addition, the variable weight functions of $T_{n}$ is defined as $\omega_{(x)}[25]$

$$
\omega_{(x)}= \begin{cases}\frac{1}{\sqrt{1-x^{2}}}, & |x|<1 \\ 0, & |x| \geq 1 .\end{cases}
$$

Mathematically, the Chebyshev wavelet arguments are defined as

$$
\begin{aligned}
\Psi_{n, m} & =\Psi(k, n, m, t), \\
n=1,2, \ldots, 2^{k-1}, \quad m & =0,1, \ldots, M-1, \quad k=1,2,3, \ldots,
\end{aligned}
$$

where positive integer $k$ denotes the value of transition, $t$ indicates the time, $m$ is degree of Chebyshev polynomials for the first kind, and $n$ denotes the considered scale of wavelet. Chebyshev wavelets are formulated with substituting the first kind $T_{n}$ with relevant weight functions for each scale and transition in (11) as follows [22, 25]:

$$
\begin{aligned}
& \psi_{m}(t) \\
& = \begin{cases}\left(2^{k / 2}\right) \times T_{m}\left(2^{k}(t)-2 n+1\right), & \frac{n-1}{2^{k-1}}<t \leq \frac{n}{2^{k-1}}, \\
0 & \text { Otherwise }\end{cases}
\end{aligned}
$$

where $T_{m}$ in (15) is obtained as

$$
T_{m}(t)= \begin{cases}\frac{1}{\sqrt{\pi}}, & m=0, \\ T_{m} \times \sqrt{\frac{2}{\pi}}, & m>0 .\end{cases}
$$

Thus, weight functions corresponding to different scales are obtained as

$$
\omega_{n}(t)=\omega\left(2^{k} \cdot t-2 n+1\right) .
$$

Regarding the idea of SM method $D$ is number of partitions in the global time, $M$ is the order of Chebyshev polynomials that is derived as

$$
2^{k-1} M=D \Longrightarrow M=\frac{D}{2^{k-1}} .
$$

Numerically, the signal $f(t)$ can be expanded with Chebyshev wavelets as $[19,22]$

$$
f(t) \approx \sum_{n=1}^{2^{k-1}} \sum_{m=0}^{M-1} C_{n, m} \psi_{n, m}(t)=C^{T} \cdot \psi(t) .
$$

Here, $C$ and $\psi(t)$ are obtained as two vectors:

$$
C=\left[c_{1}, c_{2}, c_{3}, \ldots, c_{2^{k-1}}\right]_{2^{k-1} M \times 1}^{T},
$$

where

$$
C_{i}=\left[c_{i 0}, c_{i 1}, c_{i 2}, \ldots, c_{i, M-1}\right]^{T} .
$$

Chebyshev coefficients matrix is defined as

$$
\psi(t)=\left[\psi_{1}, \psi_{2}, \psi_{3}, \ldots, \psi_{2^{k-1}}\right]_{2^{k-1} M \times 1}^{T},
$$


where

$$
\psi_{i}(t)=\left[\psi_{i 0}(t), \psi_{i 1}(t), \psi_{i 2}(t), \ldots, \psi_{i, M-1}(t)\right]^{T},
$$

where $i=1,2,4, \ldots, 2^{(k-1)}$.

Local time $t_{l}$ for collocation points are considered as follows [25]:

$$
t_{l}=\left(\frac{1}{2^{k-1} M}\right)(l-0.5), \quad l=1,2,3, \ldots, 2^{k-1} M .
$$

Subsequently, a $D \times D$-dimensional square matrix of $\phi_{n, m}(t)$ is derived as

$$
\phi_{n, m}(t)=\left[\begin{array}{lllll}
\psi\left(t_{1}\right) & \psi\left(t_{2}\right) & \cdots & \psi\left(t_{i}\right)
\end{array}\right]_{2^{k-1} M \times 2^{k-1} M} .
$$

For instance, assumption of $M=4$ and $k=2$; lies on the first four equations of Chebyshev wavelet corresponding to eight collocation points (in referring to the SM method). Accordingly, coefficient matrix of free-scaled Chebyshev wavelet of $\phi_{8,8}(t)$ corresponding to $2 M$ local times is improved as follows:

$$
\begin{aligned}
& \phi_{D \times D}
\end{aligned}
$$

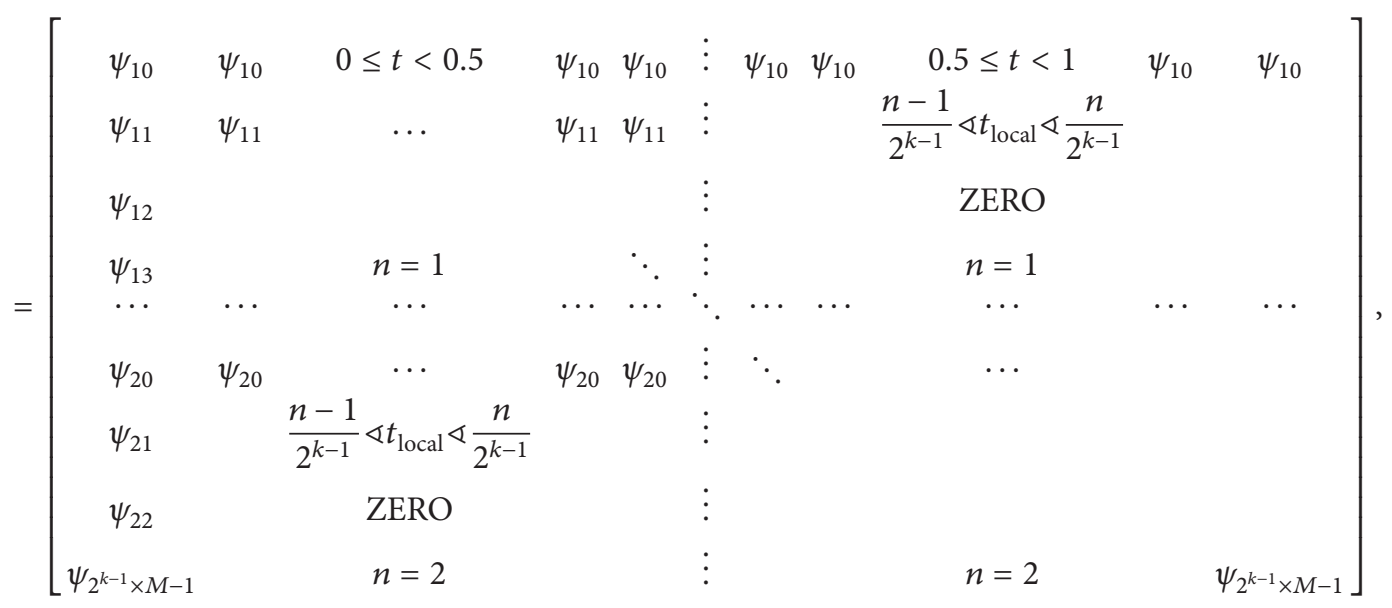

where

$$
\begin{gathered}
\text { in } \psi_{n, m} \longrightarrow n=1,2, \ldots, 2^{k-1}, \\
D=2^{k-1} \times 1 .
\end{gathered}
$$

Local times $\left(t_{l}\right)$ for $M=4$ and $k=2$ are calculated on $2 M=8$ points as

$$
t_{l}=[0.0625 ; 0.1875 ; 0.3125 ; 0.4375 ; 0.5625 ; 0.6875 ;
$$$$
0.8125 ; 0.9375] \text {. }
$$

As can be seen from the matrix of (26), column of $i$ th refers to $\psi_{i}$ and integration of $\psi(t)$ in the local time is obtained as

$$
\int_{0}^{t} \psi(\tau) d \tau \approx P \cdot \psi(t)
$$

where $D \times D$-dimensional $P$ and $\tau$ denote the operation matrix of integration and local time, respectively. Accordingly, operation matrices of integration [24] are improved as follows:

$$
\begin{aligned}
F^{\alpha}= & \frac{1}{m^{\alpha} \cdot \Gamma \alpha+2} \\
& \times\left[\begin{array}{ccccc}
a & \xi_{1} & \xi_{2} & \cdots & \xi_{2^{k-1} M-1} \\
& a & \xi_{1} & \cdots & \xi_{2^{k-1} M-2} \\
& a & \cdots & \xi_{2^{k-1} M-3} \\
& & & \\
\text { Symetric } & & \ddots & \vdots \\
& & & & \xi_{2^{k-1} M}
\end{array}\right]_{2^{k-1} M \times 2^{k-1} M}
\end{aligned}
$$

where

$$
\Gamma(\alpha+1)=\alpha !, \quad \alpha=0,1,2,3, \ldots
$$

And, respectively,

$$
\xi_{k}=(k+1)^{\alpha+1}-2 k^{\alpha+1}+(k-1)^{\alpha+1}, \quad a=\frac{\alpha+1}{2} .
$$

Hence, the operation matrix of integration is obtained as

$$
P^{\alpha}=\phi \times F^{\alpha} \times \phi^{-1}
$$




\section{Dynamic Analysis of Equation of Motion}

3.1. Haar Wavelet. Theoretically, (1) reveals one major shortcoming of Haar wavelet where at the point of 0.5 there is no continuity. In other words, the second derivation is not existed at this point. As a result, it is not possible to use this simple wavelet function, directly. Consequently, to utilize free scale of Haar wavelet functions for solution of second-ordered $O D E$ of motion, an alternative procedure is implemented, indirectly.

After discretization of external loading $F(t)$ to the $n$ equal partitions, the dynamic equilibrium governing on corresponding mass $\left(m^{*}\right)$, damping $\left(c^{*}\right)$, and stiffness $\left(k^{*}\right)$ is converted to the local time analysis as follows (related to the $2 M$ points of SM method) [16]:

$$
\begin{gathered}
\ddot{u}(t)+\frac{c^{*}}{m^{*} \cdot d_{t}} \cdot \dot{u}(t)+\frac{k^{*}}{m^{*} \cdot d_{t}} \cdot u(t) \\
=\frac{1}{m^{*} \cdot d_{t}} \cdot F\left(t_{n}+d_{t} \tau\right),
\end{gathered}
$$

where terms of velocity and acceleration are considered as

$$
\begin{gathered}
\dot{u}=d_{t} \cdot v, \\
\ddot{u}(t)=-\frac{c^{*}}{m^{*} \cdot d_{t}} \cdot \dot{u}(t)-\frac{k^{*}}{m^{*} \cdot d_{t}} \cdot u(t) \\
+\frac{1}{m^{*} \cdot d_{t}} \cdot F\left(t_{n}+d_{t} \tau\right) .
\end{gathered}
$$

Or equivalently are expanded as

$$
\begin{aligned}
& v=b P H+v_{n} E=a H, \\
& \dot{u}=d_{t} b P H+d_{t} v_{n} E=a H, \\
& u=a P H+u_{n} E .
\end{aligned}
$$

Here $a$ and $b$ are row vectors with dimension of $2 M$, for multiplying with $a P H$ or $b P H$ a unit vector is suffixed as $E_{1 \times 2 M}$. Finally, $u_{n}, v_{n}$ are initial and boundary conditions at $t=t_{n}$, that are obtained with linear interpolation of $u_{1}$ in the current interval and $u_{2 M}$ calculated from previous interval. $2 M \times 2 M$-dimensional $P$ and $H$ also represent operation of integral matrix of Haar and coefficient matrix of Haar relevant to the collocation points, respectively. Assumption of $Y=E H^{-1}$, it gives vector of $a_{1 \times 2 M}$ as [16]

$$
a=d_{t} b P+d_{t} v_{n} Y
$$

where $E=[1,1, \ldots, 1]_{1,2 M}$.
Substituting (38) into (37) vector of $b_{1 \times 2 M}$ is developed as

$$
\begin{aligned}
b_{1,2 M} & {\left[-\left(\frac{k^{*} \cdot d_{t}^{2} \cdot v_{n} \cdot Y \cdot P}{m^{*}}\right)-\left(\frac{c^{*} \cdot d_{t} \cdot v_{n}+k^{*} \cdot d_{t} \cdot v_{n}}{m^{*}}\right)\right.} \\
& \left.\cdot Y+\frac{f_{0} \cdot f t_{n}+d_{t} \tau \cdot d_{t} \cdot H^{-1}}{m^{*}}\right] \ldots \\
\times & {\left[\frac{k^{*} \cdot d_{t}^{2} \cdot P^{2}}{m^{*}}+I+\frac{c^{*} \cdot d_{t} \cdot P}{m^{*}}\right]^{-1} . }
\end{aligned}
$$

Here $I$ denotes $2 M$-dimensional identity matrix. Finally, substituting (38) and (39) into (36) and (37) vectors of acceleration, velocity, and displacement being calculated in each collocation point. A schematic view of results calculated with Haar wavelet is depicted in Figure 1. This figure shows a stairsshaped plot for responses corresponding to all collocation points of Haar wavelet.

3.2. Chebyshev Wavelet. A linear dynamic system can be expressed as

$$
m^{*} \ddot{u}(t)+c^{*} \dot{u}(t)+k^{*} u(t)=F(t) .
$$

According to (19) accelerations are numerically approximated with Chebyshev wavelet as follows:

$$
\ddot{u}(t)=C^{T} \times \psi(t) .
$$

Vector of velocity is therefore approximated multiplying by operation of integration $(P)$, as follows:

$$
\dot{u}(t)=C^{T} \times P \times \psi(t)+v_{n} .
$$

Next, displacements are numerically expanded as well as the following:

$$
u(t)=C^{T} \times P^{2} \times \psi(t)+u_{n}
$$

where, in (42) and (43), $v_{n}$ and $u_{n}$ represent initial condition of each global time, approximated with Chebyshev wavelet as a constant value. For this purpose, the unity is being expanded by the Chebyshev wavelet as [25]

$$
\begin{aligned}
1 & \cong I^{*} \times \psi(t) \\
& \cong\left(\frac{\sqrt{\pi}}{2}\right) \times[1,0,0, \ldots, 1,0,0, \ldots] \times \psi(t) .
\end{aligned}
$$

Therefore, initial displacements and velocities are improved as

$$
\begin{aligned}
& v_{n}=S_{1}^{T} \times \psi(t), \\
& u_{n}=S_{2}^{T} \times \psi(t),
\end{aligned}
$$


where $S_{1}^{T}$ and $S_{2}^{T}$ are $2^{k-1} M \times 1$ dimension vectors that are obtained by Chebyshev wavelet as follows:

$$
\begin{aligned}
& S_{1}^{T} \cong v_{n(0)} \times\left(\frac{\sqrt{\pi}}{2}\right) \times[1,0,0, \ldots, 1,0,0, \ldots]^{T}, \\
& S_{2}^{T} \cong u_{n(0)} \times\left(\frac{\sqrt{\pi}}{2}\right) \times[1,0,0, \ldots, 1,0,0, \ldots]^{T} .
\end{aligned}
$$

Substituting (45) into (42) and (43), vectors of velocity and displacement are defined as

$$
\begin{aligned}
& \dot{u}(t)=C^{T} \times P \times \psi(t)+S_{1}^{T} \times \psi(t), \\
& u(t)=C^{T} \times P^{2} \times \psi(t)+S_{1}^{T} \times P \times \psi(t)+S_{2}^{T} \times \psi(t) .
\end{aligned}
$$

Additionally, external excitation is approximated with Chebyshev wavelet as

$$
F(t)=f^{T} \times \psi(t)
$$

Equivalently, the coefficient matrix of load is numerically obtained by

$$
f_{1 \times 2^{k-1} M}^{T}=\frac{F_{1 \times 2^{k-1} M}}{\phi_{\left(2^{k-1} M\right) \times\left(2^{k-1} M\right)}} .
$$

Furthermore, quantities of dynamic system are modified corresponding on local times as

$$
\begin{aligned}
& \dot{u}(t)=d_{t} \cdot v, \\
& \ddot{u}(t)=d_{t} \cdot F\left(t_{n}+d_{t} \cdot \tau, u, v\right) .
\end{aligned}
$$

Finally, rearranging (40), after algebraic simplifications the dynamic equilibrium is numerically developed as following algebraic system:

$$
\begin{aligned}
m^{*} \cdot & {\left[C^{T}\right]+c^{*} \cdot d_{t} \cdot\left[C^{T} P+S_{1}^{T}\right] } \\
& +k^{*} \cdot d_{t}^{2} \cdot\left[C^{T} P^{2}+S_{1}^{T} P+S_{2}^{T}\right]=f^{T} d_{t}^{2} .
\end{aligned}
$$

Equation (51) represents an algebraic system, calculating $C^{T}$ and substituting into (42) and (43), displacements and velocities are obtained at any time instant, respectively.

A schematic view of responses computed with Chebyshev wavelet is depicted in Figure 1. This figure shows a linear plot for responses corresponding to all collocation points of Chebyshev wavelet.

\section{Stability Analysis}

In general, displacements, velocities, and accelerations have been transferred step-by-step from $t$ th step to the $(t+\Delta t)$ th step of integration schemes. In other words, the relation between quantities from previous state to the current state is expressed as follows [25]:

$$
\left\{\widehat{U}_{t+\Delta t}\right\}=[A]\left\{\widehat{U}_{t}\right\}+[L]\left\{\widehat{f}_{t+v}\right\}
$$

where, in (52), $\widehat{U}_{t+\Delta t}$ represents solution quantities that have been transferred with amplification matrix of $A$ from those on the previous step of $\widehat{U}_{t} ; L$ is load operators to relate external load $\widehat{f}_{t+v}$ to the current quantities, known as $\widehat{U}_{t+\Delta t} ; v$ stands on coefficient of $\Delta t$ and may be $0, \Delta t$ or $\theta \Delta t$ related to each numerical integration method.

To determine the stability criterion of the proposed scheme for Haar wavelet, the behavior of numerical integration should be examined for any initial conditions. For this purpose, the dynamic equilibrium governing on a free vibrated SDOF system in (40) is evaluated. Accordingly, (40) is rearranged in terms of damping ratio $(\xi)$ and natural frequency $(\omega)$ as

$$
\ddot{u}(t)+2 \xi \omega \dot{u}(t)+\omega^{2} u(t)=0 .
$$

Equation (39) and therefore (38) are rearranged for the initial velocity $\left(v_{n}=\dot{u}^{t-\Delta t}\right)$ as follows:

$$
\begin{aligned}
& b=\dot{u}^{t-\Delta t}\left(\kappa \mu^{-1}\right), \\
& a=\dot{u}^{t-\Delta t} \eta,
\end{aligned}
$$

where

$$
\begin{aligned}
& \kappa=-\omega^{2}(\Delta t) Y-2 \xi \omega(\Delta t) Y-\omega^{2}(\Delta t)^{2} Y P, \\
& \mu=2 \xi \omega(\Delta t) P+I+\omega^{2}(\Delta t)^{2} P^{2}, \\
& \eta=(\Delta t) \kappa \mu^{-1} P+(\Delta t) Y .
\end{aligned}
$$

Note that $\mu$ and $I$ (identity matrix) are $2 M \times 2 M$ dimensional matrix; $\kappa$ and $\eta$ are $1 \times 2 M$-dimensional vectors, respectively. Equivalently, (52) when no load is applied $\left(\widehat{f}_{t+v}=0\right)$ is developed as

$$
\left[\begin{array}{c}
\ddot{u}^{t} \\
\dot{u}^{t} \\
u^{t}
\end{array}\right]=\left[\begin{array}{ccc}
0 & d^{\prime}+e^{\prime} & c^{\prime} \\
0 & a^{\prime} & 0 \\
0 & b^{\prime} & g^{\prime}
\end{array}\right]\left[\begin{array}{c}
\ddot{u}^{t-\Delta t} \\
\dot{u}^{t-\Delta t} \\
u^{t-\Delta t}
\end{array}\right] .
$$

As it is shown in Figure 1, Haar wavelet functions calculate constant responses on all $2 M$ collocation points. Consequently, components of amplification matrix of $A$ computed in (56) represent mean value of $2 M$ points in each time interval and we have

$$
\begin{aligned}
& a^{\prime}=\operatorname{mean}\left(\kappa \mu^{-1} P H_{(t)}+E\right), \\
& b^{\prime}=\operatorname{mean}\left(\eta P H_{(t)}\right), \\
& g^{\prime}=\operatorname{mean}(E)=1, \\
& d^{\prime}=\operatorname{mean}\left(-2 \xi \omega\left(\kappa \mu^{-1} P H_{(t)}+E\right)\right), \\
& e^{\prime}=\operatorname{mean}\left(-\eta P H_{(t)} \omega^{2}\right), \\
& c^{\prime}=\operatorname{mean}\left(-E \omega^{2}\right) .
\end{aligned}
$$

Figure 1 and (56) demonstrate that responses in each time interval have been independently calculated by solver of Haar 


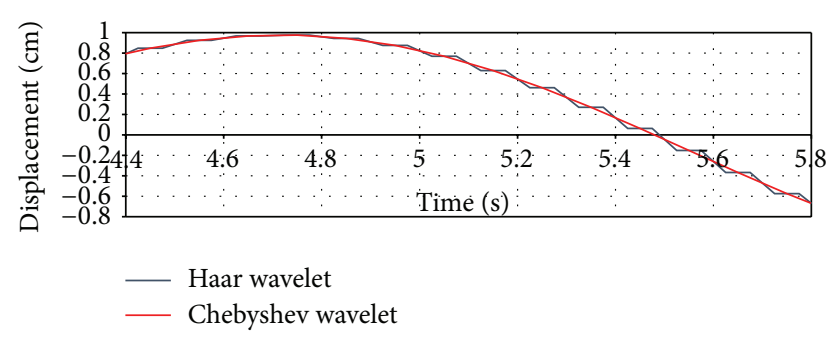

FIGURE 1: A schematic view of results calculated with wavelet functions.

from initial accelerations. Although, the same behavior is reported for Chebyshev wavelet [25], the amplification matrix of $A$ and (52) is modified for Chebyshev wavelet in order to be dependent on initial accelerations as

$$
\left[\begin{array}{c}
\dddot{x}^{t} \\
\ddot{x}^{t} \\
\dot{x}^{t} \\
x^{t}
\end{array}\right]=\left[\begin{array}{llll}
0 & a \phi(t) & b \phi(t) & c \phi(t) \\
0 & d \phi(t) & e \phi(t) & f \phi(t) \\
0 & g \phi(t) & i \phi(t) & j \phi(t) \\
0 & q \phi(t) & r \phi(t) & z \phi(t)
\end{array}\right]\left[\begin{array}{c}
\dddot{x}^{t-\Delta t} \\
\ddot{x}^{t-\Delta t} \\
\dot{x}^{t-\Delta t} \\
x^{t-\Delta t}
\end{array}\right],
$$

where

$$
\begin{aligned}
& a=\kappa \mu^{-1}, \\
& b=\eta \mu^{-1}, \\
& c=\Upsilon \mu^{-1}, \\
& d=I^{*}+\kappa \mu^{-1} P, \\
& e=\eta \mu^{-1} P, \\
& f=\Upsilon \mu^{-1} P, \\
& g=I^{*} P+\kappa \mu^{-1} P^{2}, \\
& i=I^{*}+\eta \mu^{-1} P^{2}, \\
& j=\Upsilon \mu^{-1} P^{2}, \\
& q=I^{*} P^{2}+\kappa \mu^{-1} P^{3}, \\
& r=I^{*} P+\eta \mu^{-1} P^{3}, \\
& z=I^{*}+\Upsilon \mu^{-1} P^{3},
\end{aligned}
$$

where

$$
\begin{aligned}
& \kappa=-I^{*}-2 \xi \omega(\Delta t) I^{*} P-\omega^{2}(\Delta t)^{2} I^{*} P^{2}, \\
& \eta=-2 \xi \omega(\Delta t) I^{*}-\omega^{2}(\Delta t)^{2} I^{*} P, \\
& \Upsilon=-\omega^{2}(\Delta t)^{2} I^{*}, \\
& \mu=P+2 \xi \omega(\Delta t) P^{2}+\omega^{2}(\Delta t)^{2} P^{3},
\end{aligned}
$$

where $\phi(t)$ denotes $2 M \times 2 M$-dimensional coefficient matrix of Chebyshev wavelet. $I^{*}$ and $P$ imply (44) and operation

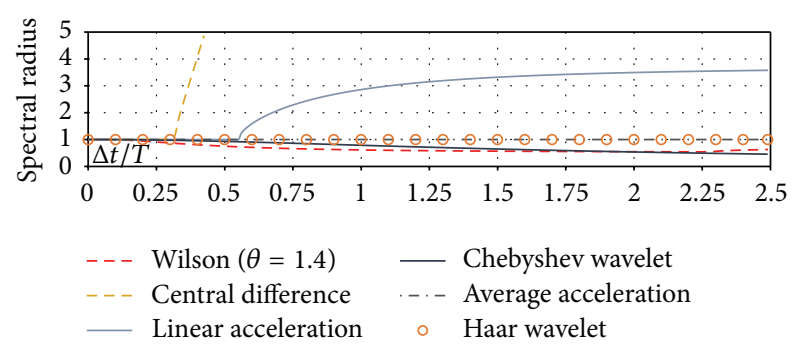

FIGURE 2: Comparison of spectral radius for proposed methods and 4 other integration schemes.

matrix of integration of Chebyshev wavelet. Note that $\mu$ is $2 M \times 2 M$-dimensional matrix; $\kappa, \eta$, and $\Upsilon$ are $1 \times 2 M$ dimensional vectors, respectively. The spectral decomposition of $A=[\Phi][\lambda][\Phi]^{-1}$ is considered for stability and $[\Phi]$ contains eigenvectors of $A$ and $[\lambda]$ is a diagonal matrix including eigenvalues of $A$. In the pursuit of stable solution the spectral radius of $A$ that equaled by maximum norm of elements $[\lambda]$ should be less than unity as follows [5]:

$$
\rho(A)=\max \left(\left\|\lambda_{1}\right\|,\left\|\lambda_{2}\right\|,\left\|\lambda_{3}\right\|\right) \leq 1,
$$

where $\rho(A)$ is the spectral radius of the amplification matrix $A$ computed as a function of $\Delta t$ by (56) for the solver of Haar or (58) for the Chebyshev wavelet. This value is calculated for some integration schemes, including, Wilson$\theta$, central difference, Newmark- $\beta$ (both linear and average acceleration integration procedure), and the second scale (the least scale) of Chebyshev and Haar wavelet. Results that have been plotted in Figure 2 based on $\xi=0$ show that the central difference method and linear acceleration method, as two explicit methods, are conditionally stable whereas the proposed scheme for simple Haar wavelet or complex Chebyshev wavelet is unconditionally stable even in the first two scales of corresponding wavelets. Therefore, no restraints (such as $\Delta t$ critical value) are placed on time step $\Delta t$ used in the analysis from the viewpoint of numerical stability considerations.

As it is shown in Figure 2, variation of spectral radius in terms of variation of $\Delta t / T$ illustrates that Wilson- $\theta$ and average acceleration method are also unconditionally stable with no requirements made on the time step $\Delta t$ used in the analysis.

\section{Numerical Applications}

In the following section validity of proposed methods is examined through the comparison of results calculated with diverse scale of Chebyshev wavelet, Haar wavelet, Duhamel method, and Wilson- $(\theta)$ method. Two examples are considered, including a fixed beam under harmonic loading and a nonlinear SDOF system under a wide-frequencycontent base acceleration. The corresponding scale of each wavelet function is designated by $2 M$. For instance, $2 M 64$ implies on 64th scale of corresponding wavelet. Furthermore, to evaluate optimal structural dynamic, computation time involved for each method is also considered, where it has been 


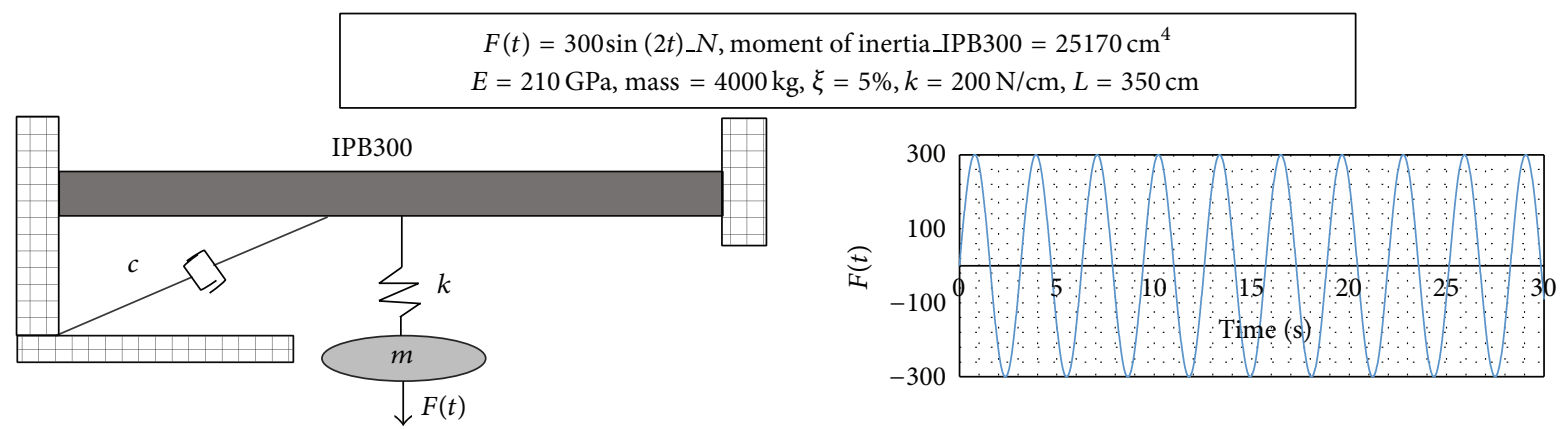

FIgURE 3: A fixed beam under sinusoidal loading $F(t)$ applied in vertical direction.

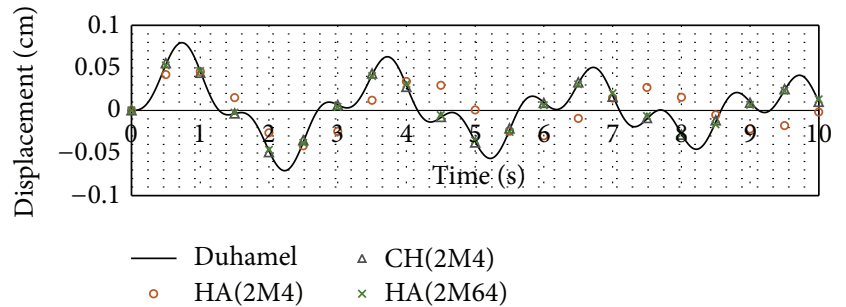

FIgURE 4: The first $10 \mathrm{sec}$ of vertical displacement of the mid node ( $\mathrm{HA}=$ Haar wavelet, $\mathrm{CH}=$ Chebyshev wavelet $)$.

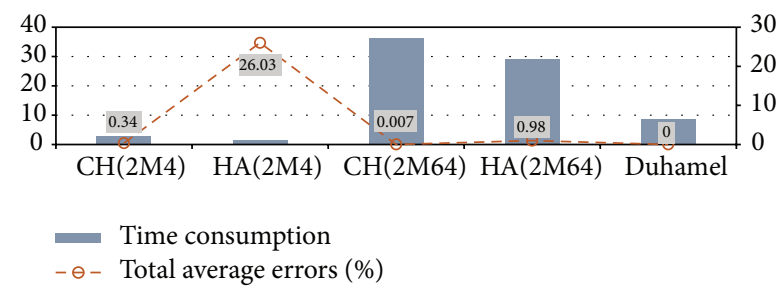

Figure 5: Computation time involved (sec) and percentile total average error for proposed methods $(\mathrm{HA}=$ Haar wavelet, $\mathrm{CH}=$ Chebyshev wavelet).

recorded with the same hardware. Similarly, for nonlinear analysis of structures recessive proceeding is developed; that is, provisions in the previous step are new initial conditions in the current step for each nonlinear behavior such as stiffness or damping of structure.

5.1. A Fixed Beam. Figure 3 shows a fixed I-shaped beam under sinusoidal loading at the center. The characteristics of considered system including section, mass, dynamic loading, damping ratio, and spring stiffness are shown in this figure. It should be noted that all degrees of freedom are neglected except vertical displacement of the center node.

The first $10 \mathrm{sec}$ time history displacement of the center node is plotted in Figure 4, calculated using the proposed methods of Haar (2M4 and 2M64) and Chebyshev wavelet (2M4), and compared with results of Duhamel method.

It can be seen from figure that accurate results are calculated by 4th scale of Chebyshev wavelet and 64th Haar wavelet. Significantly, this figure shows that result computed
TABLE 1: Absolute errors and calculation time of free-scaled Chebyshev wavelet and Duhamel (example 5.1).

\begin{tabular}{lccc}
\hline SM point & \multicolumn{2}{c}{$\begin{array}{c}\text { Chebyshev wavelet } \\
(\Delta t=0.05 \mathrm{~s})\end{array}$} & $\begin{array}{c}\text { Duhamel } \\
(\Delta t=0.01 \mathrm{~s})\end{array}$ \\
& $\Delta e$ & $t(\mathrm{sec})$ & $t(\mathrm{sec})$ \\
\hline $2 M=4$ & $3.40 E-03$ & 2.8654 & \\
$2 M=8$ & $8.91 E-04$ & 5.3475 & \\
$2 M=32$ & $1.76 E-04$ & 9.3321 & 8.527 \\
$2 M=64$ & $7.32 E-05$ & 36.3918 & \\
$2 M=128$ & $8.00 E-05$ & 78.8623 & \\
$2 M=256$ & $8.23 E-06$ & 141.1936 & \\
\hline
\end{tabular}

by 4 th scale of Haar wavelet is almost rejected but from its optimization point of view we still accept result of Haar wavelet. In addition, absolute errors and computational times due to the free scale of Chebyshev wavelet consist of 4th, 8th, 32nd, ..., 256th against Duhamel method is tabulated in Table 1 for 10 seconds of loading. It is pointed out that the long interval of $\Delta t=0.05 \mathrm{~s}$ and short time step of $\Delta t=0.01 \mathrm{~s}$ have been utilized for time increment of wavelet solver and Duhamel scheme, respectively.

Table 1 confirms that high scales of Chebyshev wavelet analyze lateral loading more accurately than low scales. However, computational time gave the maximum value of 141.19 s. Furthermore, it is shown in Table 1 that after 64th scale of Chebyshev wavelet accuracy of responses roughly remained constant. However, cost of analysis is considerably increased from the low scales to high scales of Chebyshev wavelet. For the purpose of comparison, percentile errors and computational times have been plotted in Figure 5 for two different scales of Haar and Chebyshev wavelet compared with Duhamel integration method.

It is shown in Figure 5 that cost of calculation which belongs to the 4th scale of Chebyshev wavelet gave $0.34 \%$ errors after $2.86 \mathrm{sec}$, whereas the least accuracy computed for 4th scale of Haar wavelet with $26.03 \%$ in $1.62 \mathrm{sec}$. Figure 5 shows that the cost of Duhamel method reached the third highest value of $8.52 \mathrm{sec}$. As can clearly be compared, computation time involved in the high scale of Chebyshev wavelet shown in Table 1 and Figure 5 is not desirable. In other words, for a harmonic loading known as a smooth one, the desirable responses are calculated with 4th or 8th scale of Chebyshev 


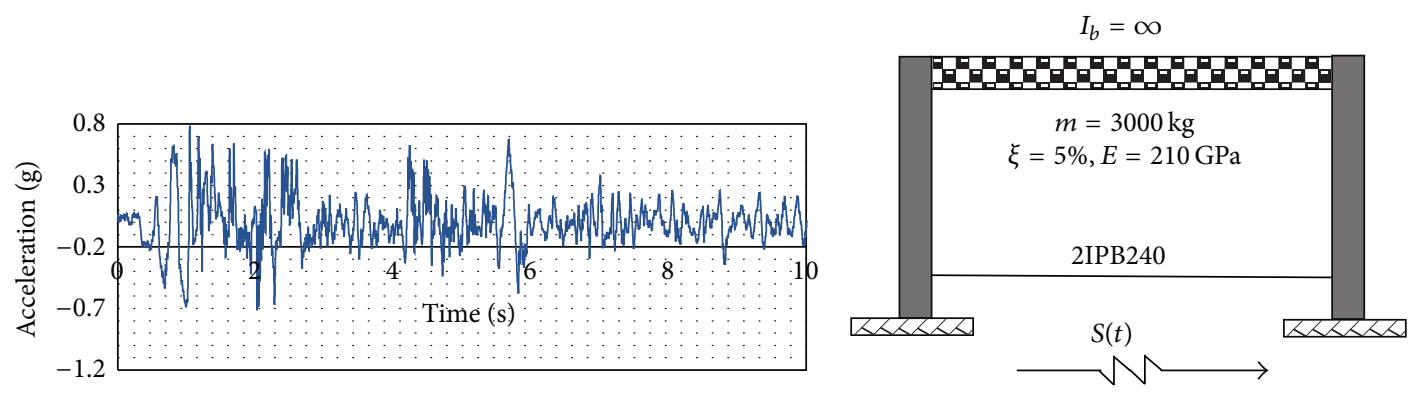

FIgURE 6: A SDOF system subjected to the El-Centro acceleration at the base.

wavelet. Overall, Duhamel results compared with responses of low scale of Chebyshev wavelet demonstrate the efficiency of this basis wavelet even for the low scale of $2 M=4$ with the least cost of computation, albeit high-scaled Chebyshev wavelet analyzes the lateral loading, accurately.

\subsection{A Nonlinear SDOF System Subjected to the Ground} Acceleration. Figure 6 shows a SDOF system subjected to ElCentro acceleration. The characteristics of considered system including two $4 \mathrm{~m}$ column's section, mass, damping ratio, and ground acceleration are shown in this figure. Nonlinearly dynamic analysis is carried out and it is assumed that the nonlinear stiffness $\left(k^{*}\right)$ is defined in term of displacement as

$$
k^{*}=\frac{4000}{\sqrt{a b s u+1}} .
$$

Nonlinear time history horizontal displacement of the considered mass using Chebyshev and Haar wavelet for time increment of $\Delta t=0.05 \mathrm{sec}$ compared with Wilson- $\theta$ method for $\Delta t=0.05$ and $0.001 \mathrm{sec}$ is plotted in Figure 7. It is shown in the figure that results computed even with low scale of Haar wavelet are closer than results calculated for a large time increment of Wilson- $\theta$. In addition, this figure illustrates high accuracy of low-scaled Chebyshev wavelet and largescaled Haar wavelet in comparison with long time increment of Wilson- $\theta$ method.

Furthermore, computation time involved and percentile total average errors are plotted in Figure 8 for proposed schemes and Wilson- $\theta$ method. For the purpose of comparison, time history responses calculated with Wilson- $\theta$ method with time increment of $\Delta t=0.001 \mathrm{sec}$ are supposed as exact results and errors are presented compared with exact responses.

Considerably, Figure 8 illustrates that optimal dynamic analysis has been achieved by 4 th scale of Chebyshev wavelet. Significantly, cost of analysis is reduced by $6.98 \mathrm{sec}$ and accuracy of $2.97 \%$ of errors. Moreover, Figure 8 shows an inefficient nonlinear analysis for Haar wavelet, particularly encountering with broad-frequency-content excitation (ElCentro acceleration shown in Figure 6). Imprecise approximation of high frequency components by Haar wavelet and using an alternative solver significantly affects the cost of analysis. Consequently, the applicability of the proposed approaches is demonstrated by given numerical examples, in which that the linear and nonlinear time history analysis of

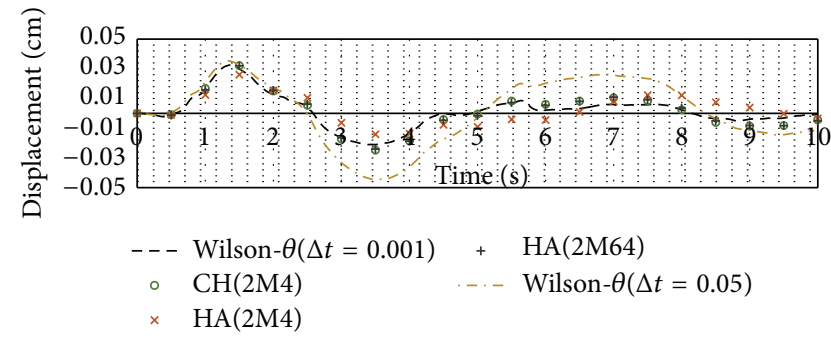

Figure 7: The first $10 \mathrm{sec}$ horizontal displacement of the SDOF system $(\mathrm{HA}=$ Haar wavelet, $\mathrm{CH}=$ Chebyshev wavelet $)$.

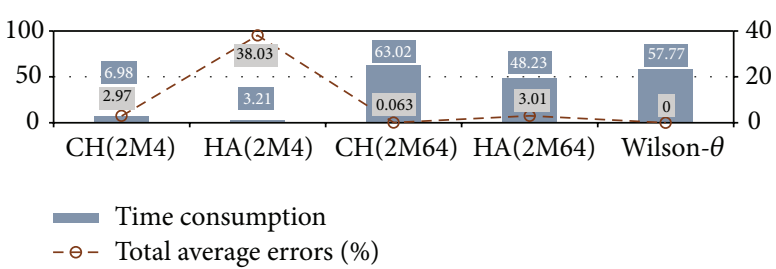

FIGURE 8: Computation time involved (sec) and percentile total average error for proposed methods; $\mathrm{HA}=$ Haar wavelet, $\mathrm{CH}=$ Chebyshev wavelet $(\Delta t=0.05)$; Wilson- $\theta(\Delta t=0.001)$.

the smooth parts and broadband frequency contents of the external loading are optimally accomplished using moderate scales of the simple Haar and low scales of the complex Chebyshev wavelets, respectively.

\section{Conclusion}

In this paper, procedure of dynamic analysis of structures is developed using wavelet functions. For this aim, implementation of two different wavelet basis functions including free-scaled complex and 3D Chebyshev and simple 2D Haar wavelet has been comparatively considered. Accordingly, for the simple and discrete shape function of Haar wavelet, an indirect solver has been applied for structural dynamics, thus computation time involved has been considerably increased, although accuracy of results was desirable for smooth loadings. Moreover, dynamic equation of motion has been analyzed through the accurate approximation of complex loading using 3D Chebyshev wavelet. It is shown that the solver of continuous Chebyshev wavelet is directly 
implemented, therefore evaluation of the equation of motion is optimally achieved. In other words, an adaptive numerical scheme has been developed to be capable of capturing details in the vicinity of highly varying structural responses. Eventually, it is demonstrated that the proposed methods for both wavelet functions lie on unconditional stable methods.

Despite accurate responses for large scales of Chebyshev wavelet due to the inherent ability of this wavelet, the optimum results were computed using its low-scaled functions. In actual fact, practice of several basis functions in the proposed approach, yielding an adaptive wavelet approach, varies from cases to cases. Consequently, compatible analysis is satisfied using 2D and simple functions to analyze the smooth parts of loading and employing 3D and complex wavelet functions to analyze wide-frequency-contents parts, simultaneously. It is recommended to employ the proposed methods for the solution of optimization problems, where the objective functions of dynamic equilibrium governing to the largescaled structural systems are considered for satisfaction of various constraints.

\section{Conflict of Interests}

The authors declare that there is no conflict of interests regarding the publication of this paper.

\section{Acknowledgments}

The authors wish to acknowledge the financial support from the University of Malaya (UM) and Ministry of Education of Malaysia (Grant nos. PG078/2013B and UM.C/625/1/HIR /MOHE/ENG/55).

\section{References}

[1] K. J. Bathe, Finite Element Procedures, Prentice-Hall, Englewood Cliffs, NJ, USA, 1996.

[2] T. J. Hughes, The Finite Element Method, Linear Static and Dynamic Finite Element Analysis, Prentice-Hall, Englewood Cliffs, NJ, USA, 1987.

[3] A. K. Chopra, Dynamic of Structures: Theory and Applications to Earthquake Engineering, Prentice Hall, Englewood Cliffs, NJ, USA, 1995.

[4] M. A. Dokainish and K. Subbaraj, "A survey of direct timeintegration methods in computational structural dynamics. I. Explicit methods," Computers \& Structures, vol. 32, no. 6, pp. 1371-1386, 1989.

[5] K. J. Bathe and E. L. Wilson, "Stability and accuracy analysis of direct integration methods," International Journal of Earthquake Engineering and Structural Dynamics, vol. 1, no. 3, pp. 283-291, 1972.

[6] S.-Y. Chang, "Explicit pseudodynamic algorithm with unconditional stability," Journal of Engineering Mechanics, vol. 128, no. 9, pp. 935-947, 2002.

[7] G. Rio, A. Soive, and V. Grolleau, "Comparative study of numerical explicit time integration algorithms," Advances in Engineering Software, vol. 36, no. 4, pp. 252-265, 2005.

[8] C. F. Chen and C. H. Hsiao, "Wavelet approach to optimizing dynamic system," IEE Proceedings-Control Theory and Applications, vol. 146, no. 2, pp. 213-219, 1999.
[9] T. Fang, X. L. Leng, and C. Q. Song, "Chebyshev polynomial approximation for dynamical response problem of random system," Journal of Sound and Vibration, vol. 266, no. 1, pp. 198206, 2003.

[10] E. Salajegheh and A. Heidari, "Time history dynamic analysis of structures using filter banks and wavelet transforms," Computers and Structures, vol. 83, no. 1, pp. 53-68, 2005.

[11] S. H. Mahdavi and H. A. Razak, "Optimum dynamic analysis of 2D frames using free-scaled wavelet functions," Latin American Journal of Solids and Structures, vol. 11, no. 6, pp. 1036-1048, 2014.

[12] N. Chen, Z. Qian, and X. Meng, "Multistep wind speed forecasting based on wavelet and gaussian processes," Mathematical Problems in Engineering, vol. 2013, Article ID 461983, 8 pages, 2013.

[13] C. Cattani, "Haar wavelets based technique in evolution problems," Proceedings of the Estonian Academy of Sciences, Physics, Mathematics, vol. 53, no. 1, pp. 45-63, 2004.

[14] L. A. Díaz, M. T. Martín, and V. Vampa, "Daubechies wavelet beam and plate finite elements," Finite Elements in Analysis and Design, vol. 45, no. 3, pp. 200-209, 2009.

[15] S. U. Islam, I. Aziz, and F. Haq, "A comparative study of numerical integration based on Haar wavelets and hybrid functions," Computers \& Mathematics with Applications, vol. 59, no. 6, pp. 2026-2036, 2010.

[16] S. H. Mahdavi and S. Shojaee, "Optimum time history analysis of SDOF structures using free scale of Haar wavelet," Structural Engineering and Mechanics, vol. 45, no. 1, pp. 95-110, 2013.

[17] U. Lepik, "Haar wavelet method for solving stiff differential equations," International Journal of Mathematics and Computation, vol. 14, no. 1, pp. 467-481, 2009.

[18] Ü. Lepik, "Numerical solution of differential equations using Haar wavelets," Mathematics and Computers in Simulation, vol. 68, no. 2, pp. 127-143, 2005.

[19] E. Babolian and F. Fattahzadeh, "Numerical computation method in solving integral equations by using Chebyshev wavelet operational matrix of integration," Applied Mathematics and Computation, vol. 188, no. 1, pp. 1016-1022, 2007.

[20] L. Fox and I. B. Parker, Chebyshev Polynomials in Numerical Analysis, Oxford University Press, London, UK, 1968.

[21] J. C. Mason and D. C. Handscomb, Chebyshev Polynomials, Chapman \& Hall/CRC, 2003.

[22] E. Babolian and F. Fattahzadeh, "Numerical solution of differential equations by using Chebyshev wavelet operational matrix of integration," Applied Mathematics and Computation, vol. 188, no. 1, pp. 417-426, 2007.

[23] M. Ghasemi and M. T. Kajani, "Numerical solution of timevarying delay systems by Chebyshev wavelets," Applied Mathematical Modelling, vol. 35, no. 11, pp. 5235-5244, 2011.

[24] Y. Li, "Solving a nonlinear fractional differential equation using Chebyshev wavelets," Communications in Nonlinear Science and Numerical Simulation, vol. 15, no. 9, pp. 2284-2292, 2010.

[25] S. H. Mahdavi and H. Abdul Razak, "A wavelet-based approach for vibration analysis of framed structures," Applied Mathematics and Computation, vol. 220, no. 1, pp. 414-428, 2013. 


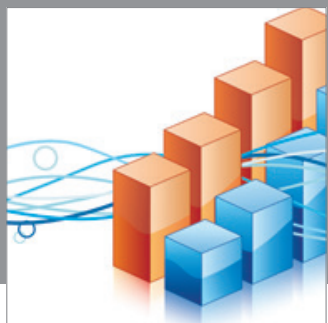

Advances in

Operations Research

mansans

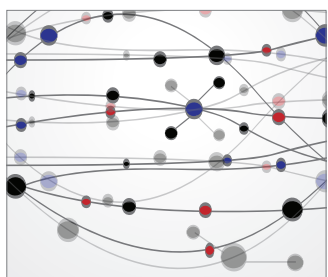

The Scientific World Journal
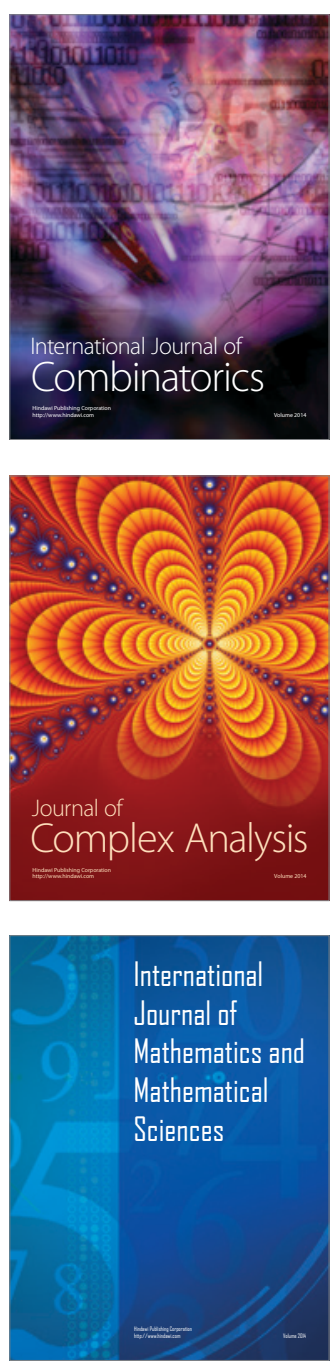
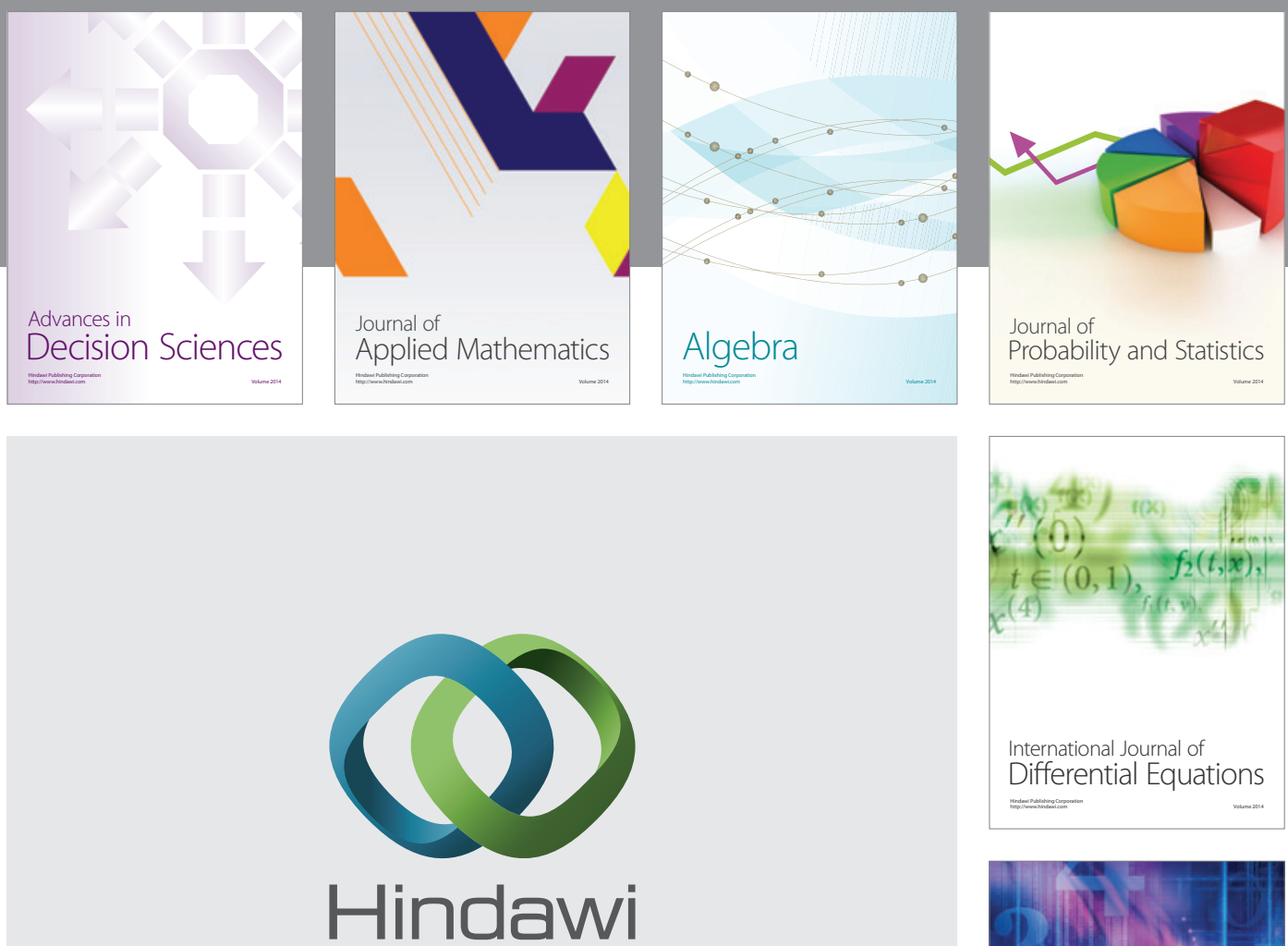

Submit your manuscripts at http://www.hindawi.com
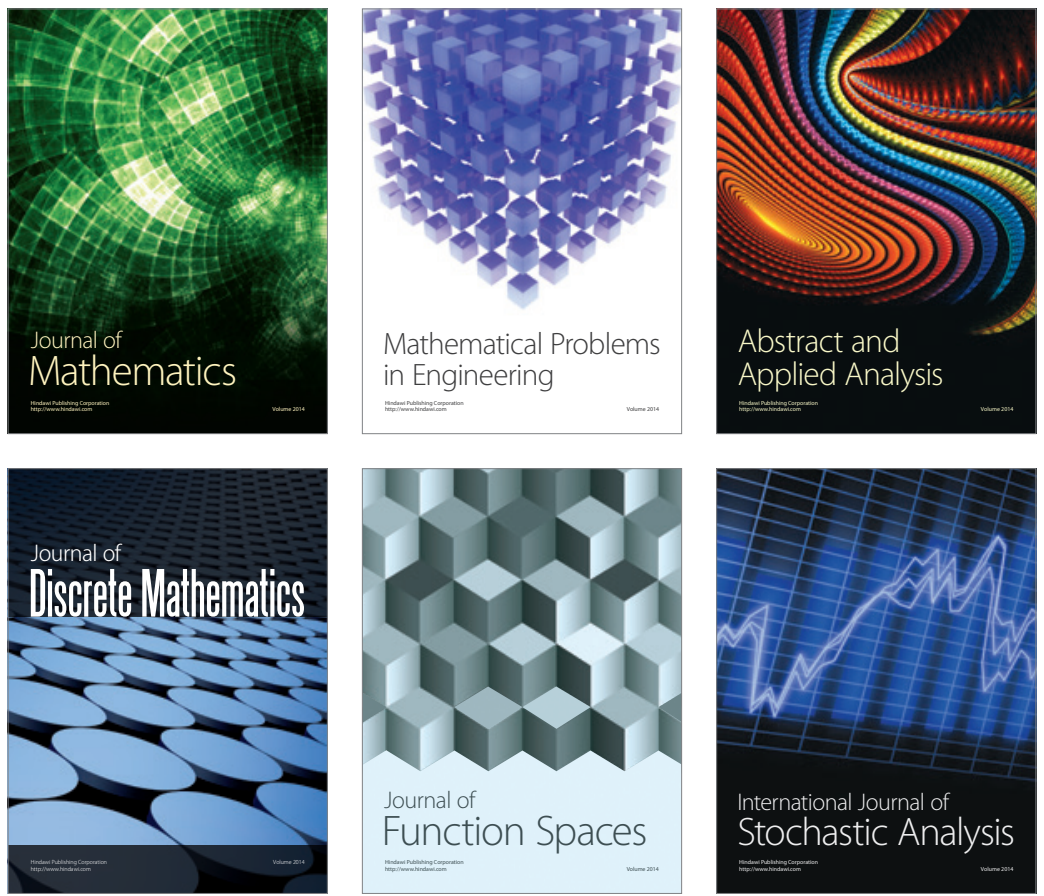

Journal of

Function Spaces

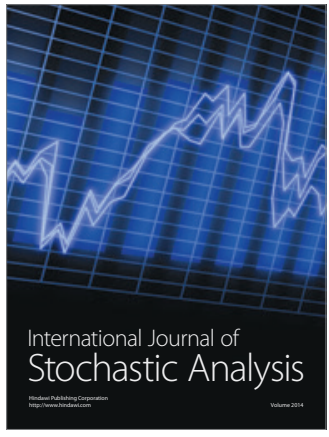

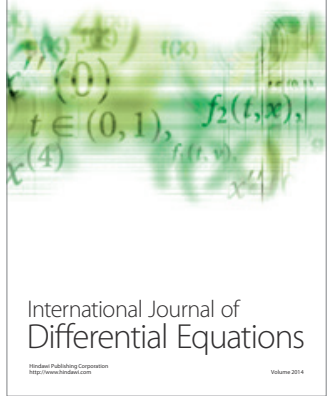
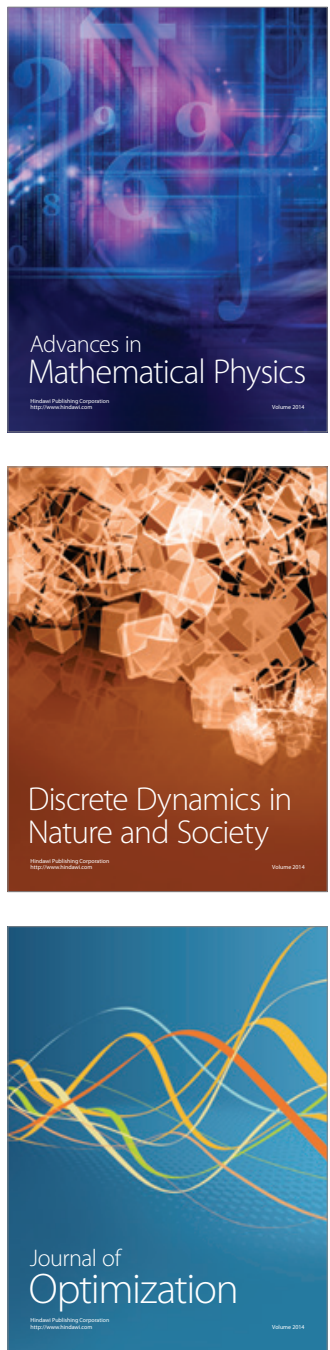